\title{
KIAA0319 and ROBO1: evidence on association with reading and pleiotropic effects on language and mathematics abilities in developmental dyslexia
}

\author{
Sara Mascheretti ${ }^{1}$, Valentina Riva ${ }^{1}$, Roberto Giorda ${ }^{2}$, Silvana Beri ${ }^{2}$, Lara Francesca Emilia Lanzoni ${ }^{1}$, \\ Maria Rosaria Cellino ${ }^{3}$ and Cecilia Marino ${ }^{4,5}$
}

Substantial heritability has been reported for developmental dyslexia (DD), and KIAAO319 and ROBO1 appear as more than plausible candidate susceptibility genes for this developmental disorder. Converging evidence indicates that developmental difficulties in oral language and mathematics can predate or co-occur with DD, and substantial genetic correlations have been found between these abilities and reading traits. In this study, we explored the role of eight single-nucleotide polymorphisms spanning within KIAAO319 and ROBO1 genes, and DD as a dichotomic trait, related neuropsychological phenotypes and comorbid language and mathematical (dis)abilities in a large cohort of 493 Italian nuclear families ascertained through a proband with a diagnosis of DD. Marker-trait association was analyzed by implementing a general test of family-based association for quantitative traits (that is, the Quantitative Transmission Disequilibrium Test, version 2.5.1). By providing evidence for significant association with mathematics skills, our data add further result in support of ROBO1 contributing to the deficits in DD and its correlated phenotypes. Taken together, our findings shed further light into the etiologic basis and the phenotypic complexity of this developmental disorder.

Journal of Human Genetics (2014) 59, 189-197; doi:10.1038/jhg.2013.141; published online 16 January 2014

Keywords: association study; developmental dyslexia; KIAAO319; language; mathematics; pleiotropy; ROBO1

\section{INTRODUCTION}

Developmental dyslexia (DD) is a complex heritable condition typically diagnosed in the first school years characterized by an impairment in reading abilities in spite of normal intelligence and adequate educational opportunities.

Converging evidence from high-risk and longitudinal studies indicates that early developmental problems in oral language ${ }^{1-7}$ and mathematical skills ${ }^{8-10}$ can constitute associated features of DD. Strong genetic correlations between language, mathematical and reading traits have been consistently reported, ${ }^{8,10-14}$ indicating that the degree of overlap in genetic influences is comparable across different regions of the liability distribution.

Even if addressing the issue of pleiotropy is among the major aims of contemporary genetic research on cognitive sciences, ${ }^{15-17}$ the identification of elements supporting shared genetic influence is a less straightforward question. Few molecular genetic studies have been conducted for language and mathematics ${ }^{18,19}$ compared with the extensive molecular genetic literature on DD. As substantial heritability has been indeed reported for DD and DD-related traits, ${ }^{15,20}$ at least nine DD loci have been mapped and specific identified genes have been consistently reported to be DD candidate susceptibility genes. ${ }^{21}$

A DD susceptibility locus on the short arm of chromosome 6, that is, DYX2, has been reported by at least five independent studies. ${ }^{21}$ From these studies of DYX2, there are two genes that stand out: KIAA0319 and DCDC2. Since the first significant association findings, ${ }^{22}$ several studies tested KIAA0319 for association with DD and DD-related traits with both positive $\mathrm{e}^{23-33}$ and negative results ${ }^{34,35}$ in clinical samples, as well as in the general population. ${ }^{36,37}$ Furthermore, a three-single-nucleotide polymorphism (SNP) risk haplotype has been shown to associate with neuropsychological skills in reading impaired families, ${ }^{21}$ likewise in general population samples. ${ }^{26,36}$ Finally, recent linkage studies reported significant findings for specific language impairment ${ }^{38,39}$ and speech sound disorder ${ }^{40}$ on this genomic region.

Linkage to the peri-centromeric region of chromosome 3 has been observed for DD susceptibility in three independent genome-

${ }^{1}$ Child Psychopathology Unit, Scientific Institute, IRCCS Eugenio Medea, Bosisio Parini, Lecco, Italy; ${ }^{2}$ Molecular Biology Lab, Scientific Institute, IRCCS Eugenio Medea, Bosisio Parini, Lecco, Italy; ${ }^{3}$ Centro Regionale di Riferimento per i Disturbi dell'Apprendimento—CRRDA, ULSS 20, Verona, Italy; ${ }^{4}$ Centre de recherche de I'Institut universitaire en santé mentale de Québec, Québec, QC, Canada and ${ }^{5}$ Département de Psychiatrie et Neurosciences, Faculté de Médecine, Université Laval, Québec, QC, Canada

Correspondence: Dr S Mascheretti, Child Psychopathology Unit, Scientific Institute, IRCCS Eugenio Medea, via don Luigi Monza, 20, 23842 Bosisio Parini (Lecco), Italy.

E-mail: sara.mascheretti@bp.Inf.it

Received 8 October 2013; revised 13 December 2013; accepted 22 December 2013; published online 16 January 2014 
wide screens. ${ }^{41}$ Hannula-Jouppi et al. ${ }^{42}$ provided the primary evidence supporting $R O B O 1$ as a DD candidate gene, reporting the observation of a translocation $\mathrm{t}(3 ; 8)(\mathrm{p} 12 ; \mathrm{q} 11)$ in an individual who also had DD, and of a $35-\mathrm{Mb}$ haplotype cosegregating with DD in the large Finnish family and including ROBO1. Recently, Bates et al. ${ }^{43}$ found associations between several SNPs of $R O B O 1$ and reading-related measures in a general population sample, indicating that $R O B O 1$ can be involved in language acquisition. A negative association between markers spanning within the ROBO1 and the diagnosis of DD have been newly found in a case-control study in an Indian sample. ${ }^{44}$ Furthermore, significant linkage results have been reported for specific language impairment ${ }^{38}$ and speech sound disorder ${ }^{45}$ to the DD locus on chromosome 3.

Finally, and perhaps of more interest, for both the risk variants mentioned hitherto, there is initial evidence of a proper functional role. In the presence of the risk haplotype on chromosome $6 \mathrm{p} 21$, the expression of the KIAA0319 gene is specifically reduced. ${ }^{46}$ This haplotype harbors the rs 9461045 minor allele, which confers reduced luciferase expression in both neuronal and non-neuronal cell lines, and is implicated in a nuclear protein-binding site. ${ }^{28}$ By knocking down the expression of this specific nuclear protein-binding site, the risk haplotype KIAA0319 expression can be almost entirely rescued. ${ }^{28}$ Functional studies have also revealed that KIAA0319 likely involved in neuronal migration and axon growth, ${ }^{47-55}$ which is in turn consistent with the original neuroanatomical finding of focal architectonic dysplasias and neuronal ectopias in the brains of people with DD. ${ }^{56,57}$ Some further support is offered by a recent animal study showing that embryonic RNA interference of KIAA0319 expression resulted in rapid auditory processing and spatial learning deficits. ${ }^{51,52}$ Regarding the ROBO1 gene, Hannula-Jouppi et al. ${ }^{42}$ demonstrated that its expression is significantly reduced by the $35-\mathrm{Mb}$ risk haplotype, which is associated with $\mathrm{DD}$ in the large Finnish family.

The bulk of results reviewed so far shows that KIAA0319 and ROBO1 genes appear as more than plausible candidates for DD, DD-related neuropsychological phenotypes and DD-correlated traits. We already yielded evidence about the effects of the other two wellreplicated DD candidate genes (that is, DYX1C1 and DCDC2) on DD and DD-related and DD-correlated phenotypes in an Italian clinical sample. ${ }^{58-60}$ In this study, we assessed the possible association with specific aspects of the phenotypic profile of DD and eight SNPs within KIAA0319-including those forming the three-SNP haplotype tested by Francks et al. ${ }^{23}$-and ROBO1, in order to further investigate the contribution of these genes to $\mathrm{DD}$ and DD-related phenotypes in an Italian sample. Moreover, we investigated the pleiotropic effects of the selected KIAA0319 and ROBO1 markers while using concurrent measurements of language and mathematics abilities.

\section{MATERIALS AND METHODS}

The protocol was approved by the Scientific Institute, IRCCS Eugenio Medea Bioethics and Scientific Board.

\section{Sample}

This study is part of an ongoing project on the genetics of reading disabilities at the Child Psychopathology Unit, Scientific Institute, IRCCS Eugenio Medea, Bosisio Parini, Lecco, Italy, and at the Centro Regionale di Riferimento per i Disturbi dell'Apprendimento-CRRDA ('Regional Reference Center for the Specific Learning Disability'), ULSS 20, Verona, Italy. ${ }^{58-64}$
The ascertainment scheme has been reported in detail elsewhere. ${ }^{61}$ Briefly, nuclear families were recruited if probands met the criteria for DD according to the DSM-IV. ${ }^{65}$ In particular, the inclusion criteria for the present study were (1) either accuracy or speed $<-2.00$ s.d. on timed text reading, single words reading or single non-word reading tests, (2) a full-scale IQ above 84 and (3) absence of neurological or sensorial disorders.

After parental informed consent, the offspring underwent an extensive medical assessment and a battery of tests, which evaluate text, word and non-word reading, ${ }^{66-68}$ writing under dictation of word, non-word and sentences-containing homophones, ${ }^{68}$ forward/backward digit ${ }^{2 p a n},{ }^{69}$ phonemic elision and blending, ${ }^{70}$ language abilities, ${ }^{71}$ mathematics abilities $^{72,73}$ and the Wechsler Intelligence Scale for Children, Revised ${ }^{74}$ or the Wechsler Intelligence Scale for Children, third edition. ${ }^{75}$ For all tests, standardized scores on the Italian population are provided (for an extensive description of tests, see Supplementary Information 1). Siblings were administered only two subtests of the intelligence scale, that is, vocabulary and block design, that show a high correlation $(r)$ with, respectively, verbal IQ $(r=0.82)^{74,75}$ and performance IQ $(r=0.73),{ }^{74,75}$ and were included if they were fully biological, older than 6 years and younger than 18 years, if they had no history of neurological and sensorial disorders and if the mean score of vocabulary and block design subtests was $>7$, regardless of their reading performance. Blood or mouthwash samples were obtained from all offspring and their biological parents.

\section{Phenotypes}

Eighteen phenotypes were used in association analyses (for a detailed description, see Supplementary Information 1): DD as a discrete trait, READING-Accuracy, READING-Speed, SPELLING, Auditory STM, ELISION, BLENDING, five language abilities (that is, semantic comprehension, Token test, syntactic comprehension, rapid automatized naming and semantic fluency) and six mathematics abilities (that is, mental calculation, both accuracy and speed; written calculation, both accuracy and speed; number dictation and numerical facts).

\section{Genotyping}

Sample collection and DNA extraction have been described in detail elsewhere. ${ }^{64}$ In particular, we explored the contribution of five among the most replicated markers within KIAA0319 and TTRAP (that is, rs2038137, rs114138463, rs4504469, rs9461045 and rs2143340) and of four markers within $R O B O 1$ (that is, rs333491, rs6803202, rs9853895 and rs7644521), which have been found associated with reading-related measures in a previous study. ${ }^{43}$ Markers rs2038136 and rs2038135 within KIAA0319 were typed as a byproduct of rs2038137 sequencing.

Portions of the region upstream of the KIAA0319 gene were amplified from genomic DNA (primer sequences and amplification protocols are available from the authors on request) and analyzed by direct sequencing. A $0.5-\mu \mathrm{l}$ aliquot of each amplified DNA sample was labeled with a BigDye Terminator 3.1 cycle sequencing kit (Life Technologies, Monza, Italy) and sequenced on an ABI3130xl Genetic Analyzer (Life Technologies). Sequences were aligned with Autoassembler (Applied Biosystems, Monza, Italy) and scored for polymorphisms rs2038137, rs2038136, rs2038135 and rs114138463. Polymorphisms rs333491, rs6803202, rs9853895 and rs7644521 in ROBO1; rs4504469 and rs9461045 in KIAA0319, and rs2143340 in TTRAP were analyzed with quantitative PCR and were typed using TaqMan SNP Genotyping assays (Life Technologies) on a 7900HT Sequence Detection System (Life Technologies).

Genotype error checking was completed in PEDSTATS, ${ }^{76}$ and inconsistent genotypes were zeroed out and were not considered for further analysis. Allelic frequencies and Hardy-Weinberg equilibrium for the markers under consideration are shown in Table 1. As SNPs required a minor allele frequency of at least $5 \%$ in order to be considered as a potential SNP for further analyses, rs114138463G/A was excluded (Table 1). For all the remaining genotyped SNPs, $P$-values for deviation from the Hardy-Weinberg equilibrium were not $<0.05$, and thus no SNPs were excluded. The linkage disequilibrium structure of KIAA0319 and ROBO1 was analyzed using the parental genotypes only; linkage disequilibrium was extracted and plotted in Haploview 4.0 
Table 1 Allele frequencies and HWE's $\boldsymbol{P}$-values for the selected KIAA0319 end ROBO1 markers

\begin{tabular}{|c|c|c|c|c|}
\hline Gene & Marker & Allele & Allele frequency ${ }^{a}$ & $H W E^{a}$ \\
\hline \multirow[t]{14}{*}{ KIAA0319 } & rs4504469c/T & C & 0.650 & 0.719 \\
\hline & & $\mathrm{T}$ & 0.350 & \\
\hline & rs2038137G/T & G & 0.663 & 0.801 \\
\hline & & $\mathrm{T}$ & 0.337 & \\
\hline & rs2038136G/C & C & 0.665 & 0.935 \\
\hline & & G & 0.335 & \\
\hline & rs2038135G/T & G & 0.678 & 0.954 \\
\hline & & $\mathrm{T}$ & 0.322 & \\
\hline & rs114138463G/A & G & 0.989 & 0.951 \\
\hline & & A & 0.011 & \\
\hline & TTRAP-rs2143340A/G & A & 0.829 & 0.999 \\
\hline & & G & 0.171 & \\
\hline & rs9461045C/T & C & 0.788 & 0.763 \\
\hline & & $\mathrm{T}$ & 0.212 & \\
\hline \multirow[t]{8}{*}{ ROBO1 } & rs6803202C/T & $\mathrm{T}$ & 0.515 & 0.992 \\
\hline & & C & 0.485 & \\
\hline & rs9853895C/T & C & 0.618 & 0.163 \\
\hline & & $\mathrm{T}$ & 0.382 & \\
\hline & rs333491A/G & A & 0.525 & 0.126 \\
\hline & & G & 0.475 & \\
\hline & $\mathrm{rs} 7644521 \mathrm{C} / \mathrm{T}$ & $\mathrm{T}$ & 0.871 & 0.991 \\
\hline & & C & 0.129 & \\
\hline
\end{tabular}

Abbreviation: HWE, Hardy-Weinberg equilibrium.

Allele frequencies and HWE were calculated in parents.

(Figures $1 \mathrm{a}$ and $\mathrm{b}$ ). Genotyped markers showing $r^{2}$-values $\geqslant 0.80$ were then dropped; on this basis, four markers for KIAA0319 gene (that is, rs4504469C/T, rs2038137G/T, rs9661045C/T and TTRAP-rs2143340A/G) and four markers for ROBO1 gene (that is, rs6803202C/T, rs9853895C/T, rs $333491 \mathrm{~A} / \mathrm{G}$ and rs7644521C/T) were then chosen for further analyses.

\section{Statistical analysis}

Genetic association was investigated by the quantitative transmission disequilibrium test (version 2.5.1) as modeled by Abecasis et al. ${ }^{77}$

Discrete trait analysis. Single-marker transmission disequilibrium test analyses were carried out for DD as a discrete trait using the '-ad' option, which allows to test using only affected individuals. As the association between the KIAA0319-selected markers and DD as a discrete trait was already investigated in a substantially overlapping sample (nuclear families' overlap $61.7 \%)^{78}$ the present study total sample, discrete trait analysis were conducted only for the ROBO1-selected SNPs.

Quantitative trait analysis. Quantitative measures were analyzed using the '-wega' option, which allows to adopt commonly used variance components, that is, the environmental variance (e), polygenic variance (g) and additive major locus (a), and the '-ao' option, which allows for families of any size (with or without parental genotypes), and should be used in a variance component framework, and represents a variant of the '-ap' option, which evaluates the evidence for population stratification. Performance IQ was included as covariate ${ }^{26,43}$ with the ' $-c$ ' option. We calculated the genetic association for the total sample for both the KIAA0319 and ROBO1 genes. Moreover, as following up association signals over different severity groups may confirm a possible true contribution of the selected SNPs to DD and DD-related performance, ${ }^{79}$ we tested the genetic association for a subsample selected by severity, that is, by selecting only the nuclear families in which at least one offspring scored $\leqslant 2.50$ s.d. below the general population mean on either accuracy or speed in either text-, or word-, or non-word reading tasks $(n=311)$. Only empirical $P$-values are reported and were computed from 1.000 Monte-Carlo permutations.
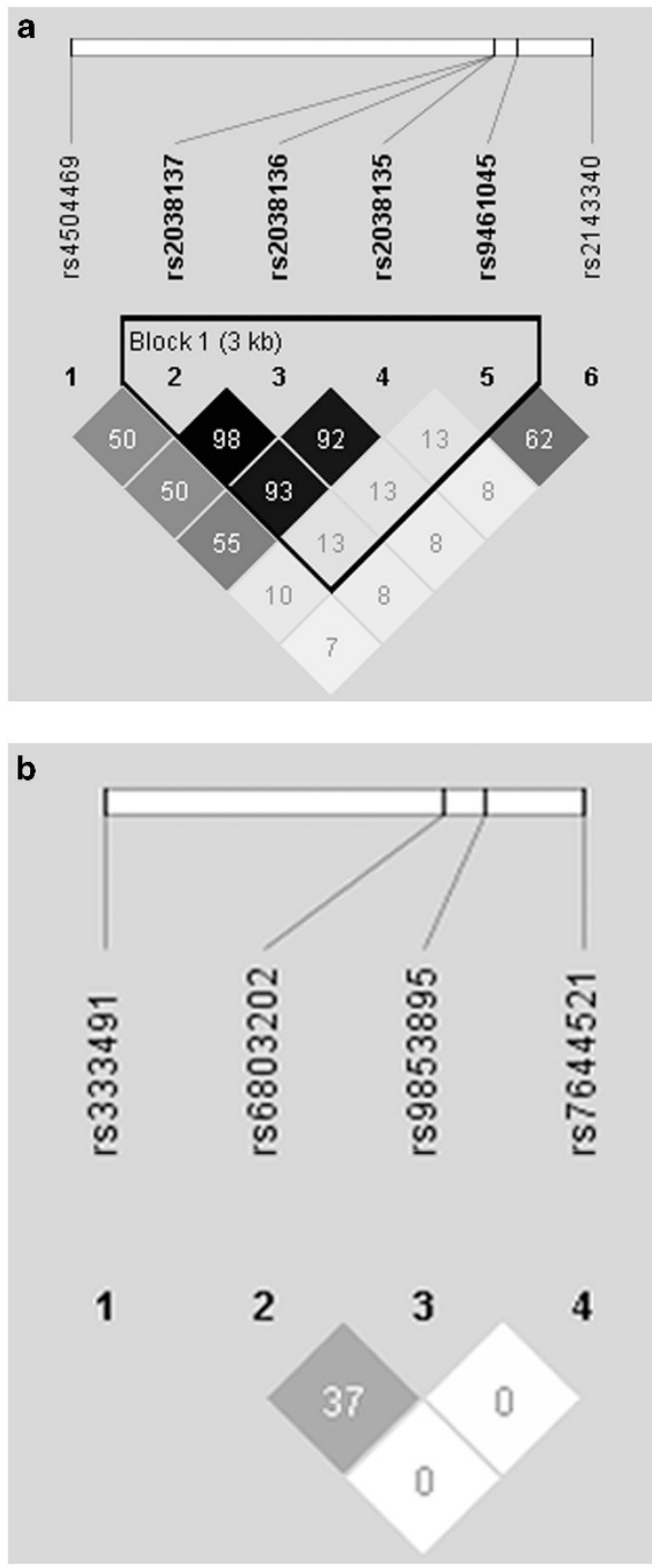

Figure 1 (a) Haploview plot showing pairwise linkage disequilibrium ( $r^{2}$-values) for seven SNPs within KIAAO319 based on parents' genotypes. Numbers in squares are $r^{2}$-values. (b) Haploview plot showing pairwise linkage disequilibrium ( $r^{2}$-values) for four SNPs within ROBO1 based on parents' genotypes. Numbers in squares are $r^{2}$-values.

The haplotype association analysis on the three-SNP haplotype ${ }^{23}$ was performed in PBAT (http://www.hsph.harvard.edu/clange/default.htm). ${ }^{80,81}$ To adjust for the covariate, we used Performance IQ as interaction variable, ${ }^{26,43}$ and nominal $P$-values are reported.

For both single-marker TDT analyses and haplotype association analysis, Bonferroni correction for multiple testing was not implemented, as it would be too conservative for multiple correlated traits (Supplementary Table 1) and non-independent genetic markers. ${ }^{23,24,34,60,82-84}$ Thus far, we decided to adjust the significance levels by the false discovery rate method $^{85}$ applied to the tests performed for each marker and haplotype (that is, 17 phenotypes), separately for each marker and haplotype. ${ }^{86-89}$ 


\section{RESULTS}

The total sample consisted of 493 unrelated nuclear families of probands with DD (804 offspring); except for 63 families that had only one parent available, all parents were represented, yielding a total sample of 1727 individuals, all of Italian ancestry. Of this total sample: (i) 352 families (599 offspring) had complete measures of READINGAccuracy, READING-Speed, SPELLING and auditory STM (group 1), and (ii) 179 families (229 offspring) had complete measures of

Table 2a Market-trait association empirical $P$-values ${ }^{\text {a }}$ in both the group 1 of the total sample $(n=352)$ and the selected-by-severity subsample $(n=311)$ for KIAA0319 markers

\begin{tabular}{|c|c|c|c|c|c|c|c|c|c|c|c|c|}
\hline & \multicolumn{9}{|c|}{ KIAA0319 marker } & \multirow{2}{*}{\multicolumn{3}{|c|}{$\begin{array}{l}\text { TTRAP marker } \\
\text { rs2143340A/G }\end{array}$}} \\
\hline & \multicolumn{3}{|c|}{ rs4504469C/T } & \multicolumn{3}{|c|}{ rs2038137G/T } & \multicolumn{3}{|c|}{ rs9661045C/T } & & & \\
\hline & $\begin{array}{c}\text { Informative } \\
\text { families }\end{array}$ & $\chi^{2}(d f)$ & $\begin{array}{l}\text { Empirical } \\
\text { P-value }\end{array}$ & $\begin{array}{c}\text { Informative } \\
\text { families }\end{array}$ & $\chi^{2}(d f)$ & $\begin{array}{c}\text { Empirical } \\
\mathrm{P} \text {-value }\end{array}$ & $\begin{array}{c}\text { Informative } \\
\text { families }\end{array}$ & $\chi^{2}(d f)$ & $\begin{array}{c}\text { Empirical } \\
\mathrm{P} \text {-value }\end{array}$ & \multicolumn{2}{|l|}{ Informative } & $\begin{array}{c}\text { Empirical } \\
\text { P-value }\end{array}$ \\
\hline \multicolumn{13}{|l|}{ READING-Accuracy } \\
\hline Total sample & 287 & $3.28(420)$ & 0.056 & 273 & $1.39(431)$ & 0.274 & 208 & $0.52(407)$ & 0.587 & 180 & $0.08(415)$ & 0.807 \\
\hline Severity sample & 235 & $2.53(351)$ & 0.112 & 228 & $1.59(360)$ & 0.224 & 182 & $0.35(344)$ & 0.632 & 157 & 0.49 (349) & 0.458 \\
\hline \multicolumn{13}{|l|}{ READING-Speed } \\
\hline Total sample & 287 & $0.13(420)$ & 0.749 & 273 & $0.05(431)$ & 0.865 & 208 & 0.14 (407) & 0.812 & 180 & $1.26(415)$ & 0.273 \\
\hline Severity sample & 235 & $0.02(351)$ & 0.903 & 228 & $0.10(360)$ & 0.787 & 182 & $0.00(344)$ & 0.999 & 157 & $2.10(349)$ & 0.145 \\
\hline \multicolumn{13}{|l|}{ SPELLING } \\
\hline Total sample & 281 & $2.07(412)$ & 0.116 & 269 & 0.02 (423) & 0.890 & 207 & 0.06 (399) & 0.856 & 180 & $1.76(407)$ & 0.247 \\
\hline Severity sample & 229 & $1.64(343)$ & 0.191 & 224 & $0.04(352)$ & 0.852 & 181 & 0.01 (336) & 0.953 & 157 & $2.27(341)$ & 0.164 \\
\hline \multicolumn{13}{|l|}{ Auditory STM } \\
\hline Total sample & 240 & $0.14(341)$ & 0.705 & 233 & 0.39 (255) & 0.588 & 168 & $0.45(331)$ & 0.458 & 149 & $0.00(340)$ & 0.944 \\
\hline Severity sample & 190 & $0.00(280)$ & 0.976 & 189 & 0.72 (290) & 0.437 & 143 & $0.34(276)$ & 0.526 & 127 & $0.00(280)$ & 0.964 \\
\hline
\end{tabular}

Table 2b Market-trait association empirical $P$-values ${ }^{\mathrm{a}}$ in both the group 1 of the total sample $(n=352)$ and the selected-by-severity subsample $(n=311)$ for $R O B O 1$ markers

\begin{tabular}{|c|c|c|c|c|c|c|c|c|c|c|c|c|}
\hline & \multicolumn{12}{|c|}{ ROBO1 } \\
\hline & \multicolumn{3}{|c|}{ rs6803202C/T } & \multicolumn{3}{|c|}{ rs9853895C/T } & \multicolumn{3}{|c|}{ rs333491A/G } & \multicolumn{3}{|c|}{ rs7644521C/T } \\
\hline & $\begin{array}{c}\text { Informative } \\
\text { families }\end{array}$ & $\chi^{2}(d f)$ & $\begin{array}{c}\text { Empirical } \\
\mathrm{P} \text {-value }\end{array}$ & $\begin{array}{c}\text { Informative } \\
\text { families }\end{array}$ & $\chi^{2}(d f)$ & $\begin{array}{c}\text { Empirical } \\
\text { P-value }\end{array}$ & $\begin{array}{c}\text { Informative } \\
\text { families }\end{array}$ & $\chi^{2}(d f)$ & $\begin{array}{c}\text { Empirical } \\
\text { P-value }\end{array}$ & $\begin{array}{c}\text { Informative } \\
\text { families }\end{array}$ & $\chi^{2}(d f)$ & $\begin{array}{c}\text { Empirical } \\
\mathrm{P} \text {-value }\end{array}$ \\
\hline \multicolumn{13}{|l|}{ READING-Accuracy } \\
\hline Total sample & 313 & $1.06(420)$ & 0.386 & 273 & 4.09 (402) & 0.110 & 270 & $0.75(410)$ & 0.366 & 173 & $1.15(407)$ & 0.218 \\
\hline Severity sample & 266 & $1.54(350)$ & 0.288 & 234 & $4.92(336)$ & 0.072 & 231 & $0.83(343)$ & 0.343 & 143 & $0.45(340)$ & 0.426 \\
\hline \multicolumn{13}{|l|}{ READING-Speed } \\
\hline Total sample & 313 & $0.55(420)$ & 0.542 & 273 & $0.00(402)$ & 0.995 & 270 & $2.74(410)$ & 0.085 & 173 & $1.22(407)$ & 0.205 \\
\hline Severity sample & 266 & $0.66(350)$ & 0.528 & 234 & 0.02 (336) & 0.880 & 231 & $1.87(343)$ & 0.142 & 143 & $0.95(340)$ & 0.236 \\
\hline \multicolumn{13}{|l|}{ SPELLING } \\
\hline Total Sample & 306 & $0.00(412)$ & 0.948 & 268 & 0.05 (394) & 0.859 & 267 & $1.24(402)$ & 0.162 & 170 & $1.30(400)$ & 0.206 \\
\hline Severity sample & 259 & $0.09(342)$ & 0.789 & 229 & 0.09 (328) & 0.815 & 228 & $1.25(335)$ & 0.186 & 140 & 1.09 (333) & 0.300 \\
\hline \multicolumn{13}{|l|}{ Auditory STM } \\
\hline Total sample & 262 & $0.23(344)$ & 0.607 & 229 & 0.02 (327) & 0.862 & 226 & $0.58(334)$ & 0.464 & 147 & $0.02(332)$ & 0.921 \\
\hline Severity sample & 220 & $0.03(281)$ & 0.846 & 195 & 0.01 (269) & 0.911 & 192 & $0.64(274)$ & 0.475 & 120 & $0.03(272)$ & 0.876 \\
\hline
\end{tabular}


ELISION, BLENDING, language and mathematics abilities (group 2; Supplementary Tables 2 and 3).

No significant associations were found between DD as a discrete trait and any of the ROBO1 markers in both the total sample and the selected-by-severity subsample.

After implementing the false discovery rate correction, market-trait associations for DD-related neuropsychological phenotypes (that is, READING-Accuracy, READING-Speed, SPELLING and Auditory
STM) were not-significant in both group 1 and in the selected-byseverity subsample (Tables $2 \mathrm{a}$ and $\mathrm{b}$ ). Regarding the DD-correlated neuropsychological traits, significant association was found between the 'A' allele of the ROBO1-rs333491A/G marker and mental calculation-accuracy $\left(\chi^{2}=10.24 ; \mathrm{df}=102\right.$; nominal $P$-value $=0.001$; empirical $P$-value $=0.003 ; q$-value $=0.030 ; 62$ informative families; genetic effect $=-0.746$; Table $3 b$ ) in group 2 . The same association was found in the selected-by-severity subsample; the 'A' allele of the

Table 3a Market-trait association empirical $P$-values ${ }^{\text {a }}$ in both the group 2 of the total sample $(n=179)$ and the selected-by-severity subsample $(n=154)$ for KIAA0319 markers

\begin{tabular}{|c|c|c|c|c|c|c|c|c|c|c|c|c|}
\hline & \multicolumn{9}{|c|}{ KIAA0319 marker } & \multicolumn{3}{|c|}{ TTRAP marker } \\
\hline & \multicolumn{3}{|c|}{ rs4504469C/T } & \multicolumn{3}{|c|}{ rs2038137G/T } & \multicolumn{3}{|c|}{$r s 9661045 \mathrm{C} / \mathrm{T}$} & \multicolumn{3}{|c|}{ rs2143340A/G } \\
\hline & $\begin{array}{c}\text { Informative } \\
\text { families }\end{array}$ & $\chi^{2}(d f)$ & $\begin{array}{l}\text { Empirical } \\
\mathrm{P} \text {-value }\end{array}$ & $\begin{array}{c}\text { Informative } \\
\text { families }\end{array}$ & $\chi^{2}(d f)$ & $\begin{array}{l}\text { Empirical } \\
\text { P-value }\end{array}$ & $\begin{array}{c}\text { Informative } \\
\text { families }\end{array}$ & $\chi^{2}(d f)$ & $\begin{array}{l}\text { Empirical } \\
\text { P-value }\end{array}$ & $\begin{array}{c}\text { Informative } \\
\text { families }\end{array}$ & $\chi^{2}(d f)$ & $\begin{array}{c}\text { Empirical } \\
\text { P-value }\end{array}$ \\
\hline ELISION & & & & & & & & & & & & \\
\hline $\begin{array}{l}\text { Total sample } \\
\text { Severity sample }\end{array}$ & $\begin{array}{r}113 \\
88\end{array}$ & $\begin{array}{l}0.28(169) \\
1.03(135)\end{array}$ & $\begin{array}{l}0.569 \\
0.264\end{array}$ & $\begin{array}{r}104 \\
83\end{array}$ & $\begin{array}{l}0.00(176) \\
0.04(140)\end{array}$ & $\begin{array}{l}0.940 \\
0.797\end{array}$ & $\begin{array}{l}82 \\
68\end{array}$ & $\begin{array}{l}0.00(159) \\
0.02(130)\end{array}$ & $\begin{array}{l}0.965 \\
0.850\end{array}$ & $\begin{array}{l}71 \\
58\end{array}$ & $\begin{array}{l}0.19(163) \\
0.19(131)\end{array}$ & $\begin{array}{l}0.524 \\
0.516\end{array}$ \\
\hline $\begin{array}{l}\text { BLENDING } \\
\text { Total sample } \\
\text { Severity sample }\end{array}$ & $\begin{array}{r}115 \\
90\end{array}$ & $\begin{array}{l}2.13(170) \\
3.59(136)\end{array}$ & $\begin{array}{l}0.133 \\
0.050 \\
0.635^{b}\end{array}$ & $\begin{array}{r}106 \\
85\end{array}$ & $\begin{array}{l}0.67(177) \\
0.46(141)\end{array}$ & $\begin{array}{l}0.321 \\
0.406\end{array}$ & $\begin{array}{l}84 \\
70\end{array}$ & $\begin{array}{l}0.52(160) \\
1.59(131)\end{array}$ & $\begin{array}{l}0.426 \\
0.161\end{array}$ & $\begin{array}{l}73 \\
60\end{array}$ & $\begin{array}{l}0.97(164) \\
1.49(132)\end{array}$ & $\begin{array}{l}0.283 \\
0.172\end{array}$ \\
\hline $\begin{array}{l}\text { SC } \\
\text { Total sample } \\
\text { Severity sample }\end{array}$ & $\begin{array}{l}50 \\
41\end{array}$ & $\begin{array}{l}0.06(91) \\
0.78(76)\end{array}$ & $\begin{array}{l}0.852 \\
0.403\end{array}$ & $\begin{array}{l}53 \\
47\end{array}$ & $\begin{array}{l}0.47(90) \\
0.61(76)\end{array}$ & $\begin{array}{l}0.451 \\
0.404\end{array}$ & $\begin{array}{l}42 \\
37\end{array}$ & $\begin{array}{l}1.85(83) \\
2.40(72)\end{array}$ & $\begin{array}{l}0.177 \\
0.141\end{array}$ & $\begin{array}{l}38 \\
34\end{array}$ & $\begin{array}{l}2.09(86) \\
2.37(74)\end{array}$ & $\begin{array}{l}0.158 \\
0.145\end{array}$ \\
\hline $\begin{array}{l}\text { TOKEN } \\
\text { Total sample } \\
\text { Severity sample }\end{array}$ & $\begin{array}{l}51 \\
42\end{array}$ & $\begin{array}{l}0.04(90) \\
0.47(75)\end{array}$ & $\begin{array}{l}0.834 \\
0.462\end{array}$ & $\begin{array}{l}53 \\
47\end{array}$ & $\begin{array}{l}0.31(89) \\
0.00(75)\end{array}$ & $\begin{array}{l}0.515 \\
0.721\end{array}$ & $\begin{array}{l}41 \\
36\end{array}$ & $\begin{array}{l}0.49(81) \\
0.74(70)\end{array}$ & $\begin{array}{l}0.466 \\
0.389\end{array}$ & $\begin{array}{l}37 \\
33\end{array}$ & $\begin{array}{l}0.36(84) \\
3.41(72)\end{array}$ & $\begin{array}{l}0.359 \\
0.214\end{array}$ \\
\hline $\begin{array}{l}\text { SYC } \\
\text { Total sample } \\
\text { Severity sample }\end{array}$ & $\begin{array}{l}48 \\
39\end{array}$ & $\begin{array}{l}0.06(91) \\
0.65(76)\end{array}$ & $\begin{array}{l}0.839 \\
0.481\end{array}$ & $\begin{array}{l}52 \\
46\end{array}$ & $\begin{array}{l}0.22(90) \\
1.47(76)\end{array}$ & $\begin{array}{l}0.718 \\
0.252\end{array}$ & $\begin{array}{l}40 \\
35\end{array}$ & $\begin{array}{l}0.06(82) \\
0.13(71)\end{array}$ & $\begin{array}{l}0.757 \\
0.638\end{array}$ & $\begin{array}{l}38 \\
34\end{array}$ & $\begin{array}{l}0.00(85) \\
0.00(73)\end{array}$ & $\begin{array}{l}0.993 \\
0.997\end{array}$ \\
\hline $\begin{array}{l}\text { RAN } \\
\text { Total sample } \\
\text { Severity sample }\end{array}$ & $\begin{array}{l}51 \\
42\end{array}$ & $\begin{array}{l}0.00(91) \\
0.12(76)\end{array}$ & $\begin{array}{l}0.953 \\
0.768\end{array}$ & $\begin{array}{l}53 \\
47\end{array}$ & $\begin{array}{l}1.11(90) \\
1.53(76)\end{array}$ & $\begin{array}{l}0.317 \\
0.248\end{array}$ & $\begin{array}{l}43 \\
38\end{array}$ & $\begin{array}{l}0.29(83) \\
0.76(72)\end{array}$ & $\begin{array}{l}0.555 \\
0.344\end{array}$ & $\begin{array}{l}39 \\
35\end{array}$ & $\begin{array}{l}0.82(86) \\
1.09(74)\end{array}$ & $\begin{array}{l}0.312 \\
0.255\end{array}$ \\
\hline $\begin{array}{l}\text { FLUENCY } \\
\text { Total sample }\end{array}$ & 51 & $0.25(90)$ & 0.668 & 51 & $0.21(89)$ & 0.647 & 41 & $8.27(81)$ & 0.013 & 37 & $3.04(84)$ & 0.062 \\
\hline Severity sample & 42 & $0.21(75)$ & 0.630 & 45 & $0.32(75)$ & 0.573 & 36 & $4.19(70)$ & $\begin{array}{c}0.044 \\
0.748^{b}\end{array}$ & 33 & $1.96(72)$ & 0.173 \\
\hline $\begin{array}{l}\text { MC-Accuracy } \\
\text { Total sample } \\
\text { Severity sample }\end{array}$ & $\begin{array}{l}70 \\
62\end{array}$ & $\begin{array}{l}2.11(117) \\
2.39(101)\end{array}$ & $\begin{array}{l}0.153 \\
0.110\end{array}$ & $\begin{array}{l}65 \\
58\end{array}$ & $\begin{array}{l}4.00(124) \\
5.12(107)\end{array}$ & $\begin{array}{l}0.055 \\
0.037 \\
0.212^{b}\end{array}$ & $\begin{array}{l}50 \\
47\end{array}$ & $\begin{array}{l}0.38(108) \\
0.51(96)\end{array}$ & $\begin{array}{l}0.497 \\
0.509\end{array}$ & $\begin{array}{l}45 \\
41\end{array}$ & $\begin{array}{l}0.49 \text { (109) } \\
0.47 \text { (97) }\end{array}$ & $\begin{array}{l}0.464 \\
0.481\end{array}$ \\
\hline $\begin{array}{l}\text { MC-Speed } \\
\text { Total sample } \\
\text { Severity sample }\end{array}$ & $\begin{array}{l}70 \\
62\end{array}$ & $\begin{array}{l}0.05(117) \\
0.01(101)\end{array}$ & $\begin{array}{l}0.839 \\
0.949\end{array}$ & $\begin{array}{l}65 \\
58\end{array}$ & $\begin{array}{l}0.57(124) \\
0.37(107)\end{array}$ & $\begin{array}{l}0.405 \\
0.537\end{array}$ & $\begin{array}{l}50 \\
47\end{array}$ & $\begin{array}{l}1.31(108) \\
0.56(96)\end{array}$ & $\begin{array}{l}0.355 \\
0.577\end{array}$ & $\begin{array}{l}45 \\
41\end{array}$ & $\begin{array}{l}0.44(109) \\
0.18(97)\end{array}$ & $\begin{array}{l}0.560 \\
0.728\end{array}$ \\
\hline $\begin{array}{l}\text { WC-Accuracy } \\
\text { Total sample } \\
\text { Severity sample }\end{array}$ & $\begin{array}{l}70 \\
62\end{array}$ & $\begin{array}{l}0.78(117) \\
0.33(101)\end{array}$ & $\begin{array}{l}0.389 \\
0.554\end{array}$ & $\begin{array}{l}65 \\
58\end{array}$ & $\begin{array}{l}4.04(124) \\
7.00(107)\end{array}$ & $\begin{array}{l}0.089 \\
0.028 \\
0.212^{b}\end{array}$ & $\begin{array}{l}50 \\
47\end{array}$ & $\begin{array}{l}0.00(108) \\
0.12(96)\end{array}$ & $\begin{array}{l}0.956 \\
0.739\end{array}$ & $\begin{array}{l}45 \\
41\end{array}$ & $\begin{array}{l}0.05 \text { (109) } \\
0.01 \text { (97) }\end{array}$ & $\begin{array}{l}0.866 \\
0.947\end{array}$ \\
\hline $\begin{array}{l}\text { WC-Speed } \\
\text { Total sample } \\
\text { Severity sample }\end{array}$ & $\begin{array}{l}70 \\
62\end{array}$ & $\begin{array}{l}0.22(116) \\
0.01\end{array}$ & $\begin{array}{l}0.538 \\
0.913\end{array}$ & $\begin{array}{l}65 \\
58\end{array}$ & $\begin{array}{l}0.20(124) \\
0.25(107)\end{array}$ & $\begin{array}{l}0.683 \\
0.625\end{array}$ & $\begin{array}{l}50 \\
47\end{array}$ & $\begin{array}{l}0.12 \text { (107) } \\
0.01 \text { (96) }\end{array}$ & $\begin{array}{l}0.690 \\
0.910\end{array}$ & $\begin{array}{l}45 \\
41\end{array}$ & $\begin{array}{l}0.05(108) \\
0.00(97)\end{array}$ & $\begin{array}{l}0.812 \\
0.961\end{array}$ \\
\hline $\begin{array}{l}\text { ND } \\
\text { Total sample } \\
\text { Severity sample }\end{array}$ & $\begin{array}{l}70 \\
62\end{array}$ & $\begin{array}{l}0.01(116) \\
0.02(100)\end{array}$ & $\begin{array}{l}0.888 \\
0.797\end{array}$ & $\begin{array}{l}65 \\
58\end{array}$ & $\begin{array}{l}0.04(123) \\
0.00(106)\end{array}$ & $\begin{array}{l}0.851 \\
0.988\end{array}$ & $\begin{array}{l}50 \\
47\end{array}$ & $\begin{array}{l}0.89 \text { (107) } \\
0.59(95)\end{array}$ & $\begin{array}{l}0.407 \\
0.483\end{array}$ & $\begin{array}{l}45 \\
41\end{array}$ & $\begin{array}{l}1.56(108) \\
1.24(96)\end{array}$ & $\begin{array}{l}0.265 \\
0.313\end{array}$ \\
\hline $\begin{array}{l}\text { NF } \\
\text { Total sample } \\
\text { Severity sample }\end{array}$ & $\begin{array}{l}70 \\
62\end{array}$ & $\begin{array}{l}0.40(117) \\
0.01(101)\end{array}$ & $\begin{array}{l}0.611 \\
0.949\end{array}$ & $\begin{array}{l}65 \\
58\end{array}$ & $\begin{array}{l}0.43(124) \\
0.68(107)\end{array}$ & $\begin{array}{l}0.567 \\
0.508\end{array}$ & $\begin{array}{l}50 \\
47\end{array}$ & $\begin{array}{l}1.16(108) \\
0.12(96)\end{array}$ & $\begin{array}{l}0.851 \\
0.767\end{array}$ & $\begin{array}{l}45 \\
41\end{array}$ & $\begin{array}{l}0.17 \text { (109) } \\
0.41 \text { (97) }\end{array}$ & $\begin{array}{l}0.688 \\
0.537\end{array}$ \\
\hline
\end{tabular}

Abbreviations: FDR, false discovery rate; FLUENCY, semantic fluency; MC, mental calculation; ND, number dictation; NF, numerical facts; RAN, rapid automatized naming; SC, semantic comprehension; SYC, syntactic comprehension; TOKEN, token test; WC, written calculation.

comprehension; SYC, syntactic comprehe

The $q$-values are bold if they are $\leqslant 0.05$.
${ }^{a}$ Empirical $P$-value computed from 1000 permutations.

bFDR correction's $q$-values. 
ROBO1-rs333491A/G marker was significantly associated with mental calculation-accuracy $\left(\chi^{2}=11.90 ; \mathrm{df}=89 ;\right.$ nominal $P$-value $\leqslant 0.001$; empirical $P$-value $\leqslant 0.001 ; q$-value $=0.010 ; 56$ informative families; genetic effect $=-0.458$; Table $3 b$ ).

No significant associations were found between KIAA0319 markers and any of the neuropsychological traits in both the total sample and the selected-by-severity subsample (Tables $2 \mathrm{a}$ and $\mathrm{b}$ and Tables $3 \mathrm{a}$ and $\mathrm{b}$ ).

Finally, we investigated the KIAA0319 three SNP haplotype (rs4504469, rs2038137 and rs2143340) originally identified by Francks et al. ${ }^{23}$ The 1-1-2 haplotype-where 1 is the major allele and 2 is the minor allele of each $\mathrm{SNP}^{23,47}$ — showed a frequency of $14.3 \%$ in our sample. After implementing the false discovery rate correction on the nominal $P$-values, significant association between this haplotype and READING-Accuracy was found in the selected-by-severity subsample (informative families $=108$, nominal $P$-value $=0.003$, $q$-value $=0.025)$.

\section{DISCUSSION}

This study was primarily designed to verify the reproducibility of previous findings of an involvement of the KIAA0319 and $\mathrm{ROBO} 1$ genes in $\mathrm{DD}$, in a sizable sample of Italian families ascertained

Table 3b Market-trait association empirical $P$-values ${ }^{a}$ in both the group 2 of the total sample $(n=179)$ and the selected-by-severity subsample $(n=154)$ for $R O B O 1$ markers

ROBO1 marker

\begin{tabular}{|c|c|c|c|c|c|c|c|c|c|c|c|c|}
\hline & \multicolumn{3}{|c|}{$r s 6803202 C / T$} & \multicolumn{3}{|c|}{ rs9853895C/T } & \multicolumn{3}{|c|}{$r s 333491 A / G$} & \multicolumn{3}{|c|}{ rs7644521C/T } \\
\hline & $\begin{array}{c}\text { Informative } \\
\text { families }\end{array}$ & $\chi^{2}(d f)$ & $\begin{array}{c}\text { Empirical } \\
\mathrm{P} \text {-value }\end{array}$ & $\begin{array}{c}\text { Informative } \\
\text { families }\end{array}$ & $\chi^{2}(d f)$ & $\begin{array}{c}\text { Empirical } \\
\text { P-value }\end{array}$ & $\begin{array}{c}\text { Informative } \\
\text { families }\end{array}$ & $\chi^{2}(d f)$ & $\begin{array}{c}\text { Empirical } \\
\text { P-value }\end{array}$ & $\begin{array}{c}\text { Informative } \\
\text { Families }\end{array}$ & $\chi^{2}(d f)$ & $\begin{array}{c}\text { Empirical } \\
\mathrm{P} \text {-value }\end{array}$ \\
\hline \multicolumn{13}{|l|}{ ELISION } \\
\hline Total sample & 115 & 0.02 (167) & 0.878 & 96 & $0.00(150)$ & 0.982 & 93 & 1.25 (157) & 0.197 & 74 & $1.00(154)$ & 0.343 \\
\hline Severity sample & 95 & 0.01 (132) & 0.908 & 81 & 0.00 (119) & 0.982 & 77 & 1.84 (124) & 0.125 & 57 & $0.53(121)$ & 0.501 \\
\hline \multicolumn{13}{|l|}{ BLENDING } \\
\hline Total sample & 116 & 1.45 (168) & 0.241 & 97 & $0.81(152)$ & 0.372 & 95 & 1.03 (159) & 0.272 & 74 & 0.33 (156) & 0.508 \\
\hline Severity sample & 96 & 1.56 (133) & 0.201 & 82 & $1.45(121)$ & 0.193 & 79 & 0.15 (126) & 0.677 & 57 & $0.24(123)$ & 0.546 \\
\hline \multicolumn{13}{|l|}{$S C$} \\
\hline Total sample & 72 & $0.10(90)$ & 0.781 & 44 & $0.53(74)$ & 0.570 & 59 & $0.02(79)$ & 0.886 & 32 & $0.28(78)$ & 0.581 \\
\hline Severity sample & 62 & $0.49(75)$ & 0.555 & 38 & $2.40(61)$ & 0.175 & 51 & 0.02 (67) & 0.898 & 27 & $0.40(66)$ & 0.551 \\
\hline \multicolumn{13}{|l|}{ TOKEN } \\
\hline Total Sample & 70 & $1.30(89)$ & 0.210 & 43 & $3.62(72)$ & 0.320 & 58 & $0.77(77)$ & 0.405 & 32 & $0.00(76)$ & 0.473 \\
\hline Severity sample & 60 & $2.03(74)$ & 0.168 & 37 & 1.32 (59) & 0.374 & 50 & $1.18(65)$ & 0.239 & 27 & $0.53(64)$ & 0.480 \\
\hline \multicolumn{13}{|l|}{ SYC } \\
\hline Total sample & 71 & $0.00(90)$ & 0.984 & 45 & $0.05(73)$ & 0.845 & 57 & $0.76(78)$ & 0.395 & 33 & $0.02(77)$ & 0.942 \\
\hline Severity sample & 61 & $0.01(75)$ & 0.923 & 39 & $0.10(60)$ & 0.791 & 49 & $1.22(66)$ & 0.328 & 28 & $0.00(65)$ & 0.965 \\
\hline \multicolumn{13}{|l|}{ RAN } \\
\hline Total sample & 73 & $0.11(90)$ & 0.721 & 45 & $0.27(74)$ & 0.604 & 59 & $1.18(79)$ & 0.341 & 32 & $0.01(78)$ & 0.934 \\
\hline Severity sample & 63 & $0.00(75)$ & 0.992 & 39 & $1.12(61)$ & 0.361 & 51 & $3.75(67)$ & 0.110 & 27 & $1.04(66)$ & 0.516 \\
\hline \multicolumn{13}{|l|}{ FLUENCY } \\
\hline Total sample & 71 & $0.71(89)$ & 0.408 & 44 & $4.08(72)$ & 0.059 & 58 & $0.11(77)$ & 0.731 & 31 & $1.95(76)$ & 0.173 \\
\hline Severity sample & 61 & $0.36(74)$ & 0.542 & 38 & 0.77 (59) & 0.403 & 50 & $0.14(65)$ & 0.691 & 26 & $3.81(64)$ & 0.065 \\
\hline \multicolumn{13}{|l|}{ MC-Accuracy } \\
\hline Total sample & 77 & $0.12(115)$ & 0.713 & 59 & $0.89(99)$ & 0.265 & 62 & $10.24(102)$ & $0.0030 .030^{b}$ & 42 & $0.58(100)$ & 0.457 \\
\hline Severity sample & 66 & 0.00 (99) & 0.962 & 53 & $0.70(85)$ & 0.303 & 56 & $11.90(89)$ & $<0.0010 .010^{b}$ & 37 & $1.36(87)$ & 0.214 \\
\hline \multicolumn{13}{|l|}{ MC-Speed } \\
\hline Total sample & 77 & $1.29(115)$ & 0.214 & 59 & $0.84(99)$ & 0.270 & 62 & $4.58(102)$ & $0.0410 .203^{b}$ & 42 & $0.03(100)$ & 0.838 \\
\hline Severity sample & 66 & 0.42 (99) & 0.454 & 53 & $0.42(85)$ & 0.385 & 56 & $4.22(89)$ & 0.071 & 37 & $0.10(87)$ & 0.663 \\
\hline \multicolumn{13}{|l|}{ WC-Accuracy } \\
\hline Total sample & 77 & $0.28(115)$ & 0.639 & 59 & 0.42 (99) & 0.500 & 62 & $3.07(102)$ & 0.100 & 42 & $0.10(100)$ & 0.731 \\
\hline Severity sample & 66 & $0.81(99)$ & 0.408 & 53 & $0.06(85)$ & 0.784 & 56 & $2.84(89)$ & 0.104 & 37 & $0.00(87)$ & 0.988 \\
\hline \multicolumn{13}{|l|}{ WC-Speed } \\
\hline Total sample & 77 & $0.51(114)$ & 0.450 & 59 & $1.13(98)$ & 0.127 & 62 & 0.99 (101) & 0.147 & 42 & 0.06 (99) & 0.742 \\
\hline Severity sample & 66 & 0.39 (99) & 0.465 & 53 & $0.84(85)$ & 0.331 & 56 & $0.72(89)$ & 0.347 & 37 & 0.59 (95) & 0.483 \\
\hline \multicolumn{13}{|l|}{$N D$} \\
\hline Total Sample & 77 & 0.62 (114) & 0.288 & 59 & 0.03 (98) & 0.815 & 62 & 0.01 (101) & 0.913 & 42 & 0.00 (99) & 0.955 \\
\hline Severity sample & 66 & $0.72(98)$ & 0.246 & 53 & $0.10(84)$ & 0.669 & 56 & $0.07(88)$ & 0.795 & 37 & $0.03(86)$ & 0.828 \\
\hline \multicolumn{13}{|l|}{$N F$} \\
\hline Total sample & 77 & 0.03 (115) & 0.839 & 59 & 0.01 (99) & 0.939 & 62 & $2.85(102)$ & 0.086 & 42 & $0.23(100)$ & 0.624 \\
\hline Severity sample & 66 & 0.02 (99) & 0.855 & 53 & $0.18(85)$ & 0.677 & 56 & $2.84(89)$ & 0.096 & 37 & $0.82(87)$ & 0.359 \\
\hline
\end{tabular}

Abbreviations: FDR, false discovery rate; FLUENCY, semantic fluency; MC, mental calculation; ND, number dictation; NF, numerical facts; RAN, rapid automatized naming; SC, semantic comprehension; SYC, syntactic comprehension; TOKEN, token test; WC, written calculation.

The $q$-values are bold if they are $\leqslant 0.05$.

aEmpirical $P$-value computed from 1000 permutations.

bFDR correction's $q$-values. 
for DD. We already yielded partial initial evidence about the effects of the KIAA0319 and ROBO1 genes on reading-related neuropsychological phenotypes in a gene-by-environment association analysis of quantitative DD-related phenotypes. ${ }^{64}$ We now integrate the study of these genes by exploring and confirming their contribution to DD, DD-related and DD-correlated phenotypes by association analyses. In particular, we provide evidence toward a role of one SNP in $R O B O 1$ (that is, rs333491A/G) in DD-related neuropsychological phenotypes, in a large Italian clinical sample. The associations were supported by the variant of the selected polymorphism, for which a positive association was found in previous works. ${ }^{43}$

Our data show that $R O B O 1$ gene is associated with the mathematics cognitive phenotypes (that is, mental calculation), suggesting that this gene can also account for part of the reduced mathematical skills that are observable in these children. To our knowledge, the only study that addressed a similar question ${ }^{19}$ reported several SNPs associated with mathematical skills in chromosomal regions that do not pertain to the gene examined in this hypothesis-driven study. Our result is quite surprising; a significant, even if low, correlation has been indeed observed between 'Mental Calculation' and 'Reading, accuracy', 'Spelling' and 'Elision' $(r=0.21, r=0.17$ and $r=0.21$, respectively; see Supplementary Table 1), and, more generally, $R O B O 1$ has been reported as a plausible candidate for DD, DD-related neuropsychological phenotypes and DD-correlated traits in previous studies. $^{41,42}$ Nevertheless, negative association between markers spanning within the $R O B O 1$ and $\mathrm{DD}$ has been also reported. ${ }^{44}$ Similarly, we did not find any association between $R O B O 1$ and DD, DD-related and DD-correlated phenotypes. This lack of association may be due to the low correlation between mathematics skills and DD-related and DD-correlated neuropsychological traits (see Supplementary Table 1). These results suggest that ROBO1 gene can account for not only part of the disabilities proper of DD, but also for part of the reduced mathematical skills that are observable in these children, thus following a pleiotropic mode of action. ${ }^{15-17,90}$

Turning to the results with the linguistic phenotypes, our negative results contrast with previously published studies ${ }^{38-40,45}$ that reported pleiotropic effects of DD candidate genes for linguistic traits. Similar to what we found in a previous study, ${ }^{60}$ this discrepancy, however, could be connected to the age of participants in our study and the type of tests that constitute adequate measures of linguistic skills at different ages. The studies that yielded positive results ${ }^{3-40}$ were indeed assessing phenotypes in children of a much younger age than ours and were able to test articulation skills, as it is appropriate for younger children. Our measures of language abilities may have turned out to be insensitive to association with these genes because the deficits in linguistic skills measured by the previous positive studies may undergo spontaneous catch-up with time and development, so that by assessing older children we found linguistically better functioning children for crucial phenotypes, such as 'articulation'. Moreover, this discrepancy may also be due to the tests used; our linguistic tests were simply not measuring the same types of deficits as measured by previous positive studies. ${ }^{45}$

Finally, our findings further support the effect of the main 'risk' rs4504469-rs2038137-rs2143340;1-1-2 haplotype ${ }^{23}$ in the etiology of reading abilities, ${ }^{23,26,36}$ by showing that it had a detrimental effect on Performance IQ-adjusted reading measure within the most severely affected children.

\section{Main conclusions}

This study provide evidence about the association between $\mathrm{ROBO1}$ and DD-related neuropsychological phenotypes. Moreover, these findings likely indicate shared biological and cognitive processes that underlie reading and mathematics, and represent a first evidence in favor of pleiotropic effect of the $\mathrm{ROBO} 1$ gene on reading-correlated neuropsychological skills in a sizable sample of Italian families ascertained for DD. Therefore, these data provide further evidence about the influence of this gene on the etiology of DD and its correlated neuropsychological traits, by exploring their effects in a non-English-speaking sample.

Nevertheless, our study should be viewed with some limitations in mind. First, even if by assuming a conservative estimate of heritability for each phenotype of 0.3 , for a sample of 493 nuclear families, the conditional power is $>95 \%$ for an $\alpha$-level of 0.05 (PBAT Power Calculator, http://www.biostat.harvard.edu/ fbat/pbat.htm), these data need replication in independent samples in order to evaluate the influence of these genes in non-English-speaking populations. Second, as with all analyses of complex traits, the significance of the current association findings with DD-correlated phenotypes should be interpreted cautiously until they can be replicated in other large, directly selected for specific language impairment, speech sound disorder and/or developmental dyscalculia samples.

\section{ACKNOWLEDGEMENTS}

We thank all the parents and children who took part in this study. We express our gratitude to Ermanno Quadrelli, Vittoria Trezzi, Lisa Meneghello and Elisabetta Furioni for helping in data collection.

1 Bishop, D. V. \& Adams, C. A prospective study of the relationship between specific language impairment, phonological disorders and reading retardation. J. Child. Psychol. Psychiatry 31, 1027-1050 (1990).

2 Nathan, L., Stackhouse, J., Goulandris, N. \& Snowling, M. J. The development of early literacy skills among children with speech difficulties: a test of the "critical age hypothesis". J. Speech. Lang. Hear. Res. 47, 377-391 (2004).

3 Stothard, S. E., Snowling, M. J., Bishop, D. V., Chipchase, B. B. \& Kaplan, C. A. Language-impaired preschoolers: a follow-up into adolescence. J. Speech. Lang. Hear. Res. 41, 407-418 (1998).

4 Snowling, M., Bishop, D. V. \& Stothard, S. E. Is preschool language impairment a risk factor for dyslexia in adolescence? J. Child. Psychol. Psychiatry 41, 587-600 (2000).

5 Lyytinen, P., Poikkeus, A. M., Laakso, M. L., Eklund, K. \& Lyytinen, H. Language development and symbolic play in children with and without familial risk for dyslexia. J. Speech. Lang. Hear. Res. 44, 873-885 (2001).

6 Lyytinen, P., Eklund, K. \& Lyytinen, H. Language development and literacy skills in late-talking toddlers with and without familial risk for dyslexia. Ann. Dyslexia 55, 166-192 (2001)

7 Rescorla, L. Age 13 language and reading outcomes in late-talking toddlers. J. Speech. Lang. Hear. Res. 48, 459-472 (2005).

8 Markowitz, E. M., Willemsen, G., Trumbetta, S. L., van Beijsterveldt, T. C. \& Boomsma, D. I. The etiology of mathematical and reading (dis)ability covariation in a sample of Dutch twins. Twin Res. Hum. Genet. 8, 585-593 (2005)

9 Light, J. G. \& DeFries, J. C. Comorbidity of reading and mathematics disabilities: genetic and environmental etiologies. J. Learn. Disabil. 28, 96-106 (1995).

10 Knopik, V. S. \& DeFries, J. C. Etiology of covariation between reading and mathematics performance: a twin study. Twin Res. 2, 226-234 (1999).

11 Thompson, L. A., Fagan, J. F. \& Fulker, D. W. Longitudinal prediction of specific cognitive abilities from infant novelty preference. Child Dev. 62, 530-538 (1991).

12 Hohnen, B. \& Stevenson, J. The structure of genetic influences on general cognitive, language, phonological, and reading abilities. Dev. Psychol. 35, 590-603 (1999).

13 Kovas, Y., Haworth, C. M., Harlaar, N., Petrill, S. A., Dale, P. S. \& Plomin, R. Overlap and specificity of genetic and environmental influences on mathematics and reading disability in 10-year-old twins. J. Child. Psychol. Psychiatry 48, 914-922 (2007).

14 Bishop, D. V. Genetic influences on language impairment and literacy problems in children: same or different? J. Child. Psychol. Psychiatry 42, 189-198 (2001).

15 Plomin, R. \& Kovas, Y. Generalist genes and learning disabilities. Psychol. Bull. 131, 592-617 (2005)

16 Kovas, Y. \& Plomin, R. Generalist genes: implications for the cognitive sciences. Trends Cogn. Sci. 10, 198-203 (2006).

17 Trzaskowski, M., Davis, O. S., DeFries, J. C., Yang, J., Visscher, P. M. \& Plomin, R. DNA evidence for strong genome-wide pleiotropy of cognitive and learning abilities. Behav. Genet. 43, 267-273 (2013).

18 Grigorenko, E. L. Speaking genes or genes for speaking? Deciphering the genetics of speech and language. J. Child. Psychol. Psychiatry 50, 116-125 (2009). 
19 Docherty, S. J., Davis, O. S., Kovas, Y., Meaburn, E. L., Dale, P. S., Petrill, S. A. et al. A genome-wide association study identifies multiple loci associated with mathematics ability and disability. Genes Brain Behav. 9, 234-247 (2010).

20 Fisher, S. E. \& DeFries, J. C. Developmental dyslexia: genetic dissection of a complex cognitive trait. Nat. Rev. Neurosci. 3, 767-780 (2002).

21 Scerri, T. S. \& Schulte-Korne, G. Genetics of developmental dyslexia. Eur. Child. Adolesc. Psychiatry 19, 179-197 (2010).

22 Kaplan, D. E., Gayan, J., Ahn, J., Won, T. W., Pauls, D., Olson, R. K. et al. Evidence for linkage and association with reading disability on 6p21.3-22. Am. J. Hum. Genet. 70 1287-1298 (2002)

23 Francks, C., Paracchini, S., Smith, S. D., Richardson, A. J., Scerri, T. S., Cardon, L. R et al. A 77-kilobase region of chromosome 6 p22.2 is associated with dyslexia in families from the United Kingdom and from the United States. Am. J. Hum. Genet. 75, 1046-1058 (2004)

24 Cope, N., Harold, D., Hill, G., Moskvina, V., Stevenson, J., Holmans, P. et al. Strong evidence that KIAA0319 on chromosome $6 p$ is a susceptibility gene for developmenta dyslexia. Am. J. Hum. Genet. 76, 581-591 (2005).

25 Harold, D., Paracchini, S., Scerri, T., Dennis, M., Cope, N., Hill, G. et al. Further evidence that the KIAA0319 gene confers susceptibility to developmental dyslexia. Mol. Psychiatry. 11, 1085-1091 (2006).

26 Luciano, M., Lind, P. A., Duffy, D. L., Castles, A., Wright, M. J., Montgomery, G. W. et al. A haplotype spanning KIAA0319 and TTRAP is associated with normal variation in reading and spelling ability. Biol. Psychiatry 62, 811-817 (2007).

27 Ludwig, K. U., Roeske, D., Schumacher, J., Schulte-Korne, G., Konig, I. R., Warnke, A. et al. Investigation of interaction between DCDC2 and KIAA0319 in a large German dyslexia sample. J. Neural Transm. 115, 1587-1589 (2008).

28 Dennis, M., Paracchini, S., Scerri, T. S., Prokunina-Olsson, L., Knight, J. C., WadeMartins, R. et al. A common variant associated with dyslexia reduces expression of the KIAA0319 gene. PLoS Genet. 5, e1000436 (2009).

29 Couto, J. M., Livne-Bar, I., Huang, K., Xu, Z., Cate-Carter, T., Feng, Y. et al. Association of reading disabilities with regions marked by acetylated $\mathrm{H} 3$ histones in KIAA0319. Am. J. Med. Genet. B. Neuropsychiatr. Genet. 153B, 447-462 (2010).

30 Elbert, A., Lovett, M. W., Cate-Carter, T., Pitch, A., Kerr, E. N. \& Barr, C. L. Genetic variation in the KIAA0319 5 ' region as a possible contributor to dyslexia. Behav. Genet. 41, 77-89 (2011).

31 Kirsten, H., Wilcke, A., Ligges, C., Boltze, J. \& Ahnert, P. Association study of a functional genetic variant in KIAA0319 in German dyslexics. Psychiatr. Genet. 22, 216-217 (2012).

32 Venkatesh, S. K., Siddaiah, A., Padakannaya, P. \& Ramachandra, N. B. Analysis of genetic variats of dyslexia candidate genes KIAA0319 and DCDC2 in Indian population. J. Hum. Genet. 58, 531-538 (2013).

33 Zou, L., Chen, W., Shao, S., Sun, Z., Zhong, R., Shi, J. et al. Genetic variants in KIAA0319, but not in DYX1C1, is associated with risk of dyslexia: an integrated metaamalysis. Am. J. Med. Genet. B. Neuropsychiatr. Genet. 159B, 970-976 (2012).

34 Brkanac, Z., Chapman, N. H., Matsushita, M. M., Chun, L., Nielsen, K., Cochrane, E. et al. Evaluation of candidate genes for DYX1 and DYX2 in families with dyslexia. Am. J. Med. Genet. B. Neuropsychiatr. Genet. 144B, 556-560 (2007).

35 Svidnicki, M.C., Salgado, C.A., Lima, R.F., Ciasca, S.M., Secolin, R., Pomilio, M.C. et al. Study of candidate genes for dyslexia in Brazilian individuals. Genet. Mol. Res. 12, 5356-5364 (2013).

36 Paracchini, S., Steer, C. D., Buckingham, L. L., Morris, A. P., Ring, S., Scerri, T. et al. Association of the KIAA0319 dyslexia susceptibility gene with reading skills in the general population. Am. J. Psychiatry 165, 1576-1584 (2008).

37 Scerri, T. S., Morris, A. P., Buckingham, L. L., Newbury, D. F., Miller, L. L., Monaco, A. P. et al. DCDC2, KIAA0319 and CMIP are associated with reading-related traits. Biol. Psychiatry 70, 237-245 (2011).

38 Rice, M. L., Smith, S. D. \& Gayan, J. Convergent genetic linkage and associations to language, speech and reading measures in families of probands with Specific Language Impairment. J. Neurodev. Disord. 1, 264-282 (2009).

39 Newbury, D. F., Paracchini, S., Scerri, T. S., Winchester, L., Addis, L., Richardson, A. J. et al. Investigation of dyslexia and SLI risk variants in reading- and language-impaired subjects. Behav. Genet. 41, 90-104 (2011).

40 Smith, S. D., Pennington, B. F., Boada, R. \& Shriberg, L. D. Linkage of speech sound disorder to reading disability loci. J. Child. Psychol. Psychiatry 46, 1057-1066 (2005).

41 Nopola-Hemmi, J., Myllyluoma, B., Haltia, T., Taipale, M., Ollikainen, V., Ahonen, T. et al. A dominant gene for developmental dyslexia on chromosome 3. J. Med. Genet. 38, 658-664 (2001)

42 Hannula-Jouppi, K., Kaminen-Ahola, N., Taipale, M., Eklund, R., Nopola-Hemmi, J., Kaariainen, H. et al. The axon guidance receptor gene ROBO1 is a candidate gene for developmental dyslexia. PLoS Genet. 1, e50 (2005).

43 Bates, T. C., Luciano, M., Medland, S. E., Montgomery, G. W., Wright, M. J. \& Martin, N. G. Genetic variance in a component of the language acquisition device: ROBO1 polymorphisms associated with phonological buffer deficits. Behav. Genet. 41, 50-57 (2011).

44 Venkatesh, S. K., Siddaiah, A., Padakannaya, P. \& Ramachandra, N. B. Lack of association between genetic polymorphisms in ROBO1, MRPL19/C2ORF3 and THEM2 with developmental dyslexia. Gene 529, 215-219 (2013).

45 Stein, C. M., Schick, J. H., Gerry Taylor, H., Shriberg, L. D., Millard, C., Kundtz-Kluge, A. et al. Pleiotropic effects of a chromosome 3 locus on speech-sound disorder and reading. Am. J. Hum. Genet. 74, 283-297 (2004).

46 Paracchini, S., Thomas, A., Castro, S., Lai, C., Paramasivam, M., Wang, Y. et al. The chromosome $6 \mathrm{p} 22$ haplotype associated with dyslexia reduces the expression of
KIAA0319, a novel gene involved in neuronal migration. Hum. Mol. Genet. 15, 1659-1666 (2006)

47 Velayos-Baeza, A., Levecque, C., Kobayashi, K., Holloway, Z G. \& Monaco, A. P. The dyslexia-associated KIAA0319 protein undergoes proteolytic processing with \{gamma\} secretase-independent intramembrane cleavage. J. Biol. Chem. 285, 40148-40162 (2010).

48 Peschansky, V. J., Burbridge, T. J., Volz, A. J., Fiondella, C., Wissner-Gross, Z. Galaburda, A. M. et al. The effect of variation in expression of the candidate dyslexia susceptibility gene homolog Kiaa0319 on neuronal migration and dendritic morphology in the rat. Cereb. Cortex 20, 884-897 (2010)

49 Currier, T. A., Etchegaray, M. A., Haight, J. L., Galaburda, A. M. \& Rosen, G. D. The effects of embryonic knockdown of the candidate dyslexia susceptibility gene homologue Dyx1c1 on the distribution of GABAergic neurons in the cerebral cortex. Neuroscience 172, 535-546 (2011).

50 Poon, M. W., Tsang, W. H., Chan, S. O., Li, H. M., Ng, H. K. \& Waye, M. M. Dyslexiaassociated kiaa0319-like protein interacts with axon guidance receptor nogo receptor 1. Cell. Mol. Neurobiol. 31, 27-35 (2011).

51 Szalkowski, C. E., Fiondella, C. F., Truong, D. T., Rosen, G. D., Loturco, J. J. \& Fitch, R. H. The effects of KiaaO319 knockdown on cortical and subcortical anatomy in male rats. Int. J. Dev. Neurosci. 31, 116-122 (2012).

52 Szalkowski, C. E., Fiondella, C. G., Galaburda, A. M., Rosen, G. D., Loturco, J. J. \& Fitch, R. H. Neocortical disruption and behavioral impairments in rats following in utero RNAi of candidate dyslexia risk gene Kiaa0319. Int. J. Dev. Neurosci. 30, 293-302 (2012)

53 Centanni, T. M., Engineer, C. T. \& Kilgard, M. P. Cortical speech-evoked response patterns in multiple auditory fields are correlated with behavioral discrimination ability. J. Neurophysiol. 110, 177-189 (2013).

54 Platt, M. P., Adler, W. T., Mehlhorn, A. J., Johnson, G. C., Wright, K. A., Choi, R. T. et al. Embryonic disruption of the candidate dyslexia susceptibility gene homolog Kiaa0319-like results in neuronal migration disorders. Neuroscience 248C, 585-593 (2013).

55 Adler, W. T., Platt, M. P., Mehlhorn, A. J., Haight, J. L., Currier, T. A., Etchegaray, M. A et al. Position of neocortical neurons transfected at different gestational ages with shRNA targeted against candidate dyslexia susceptibility genes. PLoS One 8, e65179 (2013).

56 Galaburda, A. M. \& Kemper, T. L. Cytoarchitectonic abnormalities in developmenta dyslexia: a case study. Ann. Neurol. 6, 94-100 (1979).

57 Galaburda, A. M., Sherman, G. F., Rosen, G. D., Aboitiz, F. \& Geschwind, N. Developmental dyslexia: four consecutive patients with cortical anomalies. Ann. Neurol. 18, 222-233 (1985).

58 Marino, C., Citterio, A., Giorda, R., Facoetti, A., Menozzi, G., Vanzin, L. et al. Association of short-term memory with a variant within DYX1C1 in developmental dyslexia. Genes Brain Behav. 6, 640-646 (2007).

59 Marino, C., Mascheretti, S., Riva, V., Cattaneo, F., Rigoletto, C., Rusconi, M. et al. Pleiotropic effects of DCDC2 and DYX1C1 genes on language and mathematics traits in nuclear families of developmental dyslexia. Behav. Genet. 41, 67-76 (2011).

60 Marino, C., Meng, H., Mascheretti, S., Rusconi, M., Cope, N., Giorda, R. et al. DCDC2 genetic variants and susceptibility to developmental dyslexia. Psychiatr. Genet. 22, 25-30 (2012).

61 Marino, C., Giorda, R., Vanzin, L., Molteni, M., Lorusso, M. L., Nobile, M. et al. No evidence for association and linkage disequilibrium between dyslexia and markers of four dopamine-related genes. Eur. Child Adolesc. Psychiatry 12, 198-202 (2003).

62 Marino, C., Giorda, R., Vanzin, L., Nobile, M., Lorusso, M. L., Baschirotto, C. et al. A locus on 15q15-15qter influences dyslexia: further support from a transmission/ disequilibrium study in an Italian speaking population. J. Med. Genet. 41, 42-46 (2004).

63 Marino, C., Giorda, R., Lorusso, L. M., Vanzin, L., Salandi, N., Nobile, M. et al. A family-based association study does not support DYX1C1 on $15 q 21.3$ as a candidate gene in developmental dyslexia. Eur. J. Hum. Genet. 13, 491-499 (2005).

64 Mascheretti, S., Bureau, A., Battaglia, M., Simone, D., Quadrelli, E., Croteau, J. et al. An assessment of gene-by-environment interactions in developmental dyslexia-related phenotypes. Genes Brain Behav. 12, 47-55 (2013).

65 American Psychiatric Association. Diagnostic and Statistical Manual of Mental Disorders - 4th edn (American Psychiatric Association, 1994).

66 Cornoldi, C. \& Colpo, G. Prove di lettura MT, nuove prove di lettura MT per la scuola media inferiore (Firenze, Italy: Organizzazioni Speciali, 1995)

67 Cornoldi, C. \& Colpo, G. Prove di lettura MT per la scuola elementare-2 (Firenze, Italy: Organizzazioni Speciali, 1998)

68 Sartori, G., Job, R. \& Tressoldi, P. E. Batteria per la valutazione della dislessia e della disortografia evolutiva (Firenze, Italy: Organizzazioni Speciali, 1995).

69 Reynolds, C. R. \& Bigler, E. D. Test of Memory and Learning (Trento, Italy: Erickson, 1994).

70 Cossu, G., Shankweiler, D., Liberman, I. Y., Katz, L. \& Tola, G. Awareness of phonological segments and reading ability in Italian children. Appl. Psycholinguist 9, 1-16 (1988).

71 Fabbro, F. Neurolinguistica e Neuropsicologia Dei Disturbi Specifici Del Linguaggio Nel Bambino: Proposta Di Un Esame Del Linguaggio. Saggi. Neuropsicologia Infantile, psicopedagogia, riabilitazione 1, 11-23 (1999).

72 Cornoldi, C., Lucangeli, D. \& Bellina, M. AC-MT, Test Di Valutazione Delle Abilità Di Calcolo-Gruppo MT (Trento, Italy: Erickson, 2003).

73 Cornoldi, C. \& Lucangeli, D. Arithmetic education and learning disabilities in Italy. J. Learn Disabil. 37, 42-49 (2004). 
74 Wechsler, D. Wechsler Intelligence Scale for Children, revised (Firenze, Italy: Organizzazioni Speciali, 1981).

75 Wechsler, D. Wechsler Intelligence Scale for Children-3rd edn (Firenze, Italy: Organizzazioni Speciali, 2006).

76 Wigginton, J. E. \& Abecasis, G. R. PEDSTATS: descriptive statistics, graphics and quality assessment for gene mapping data. Bioinformatics 21, 3445-3447 (2004).

77 Abecasis, G. R., Cardon, L. R. \& Cookson, W. O. A general test of association for quantitative traits in nuclear families. Am. J. Hum. Genet. 66, 279-292 (2000).

78 Eicher, J. D., Powers, N. R., Miller, L. L., Mueller, K. L, Marino, C., Smith, S. D. et al. Characterization of the DYX2 locus on chromosome 6p22 with reading disability, language impairment, and overall cognition. (in press).

79 Ludwig, K. U., Roeske, D., Herms, S., Schumacher, J., Warnke, A. \& Plume, E. Variation in GRIN2B contributes to weak performance in verbal short-term memory in children with dyslexia. Am. J. Med. Genet. B. Neuropsychiatr. Genet 153, 503-511 (2009).

80 Lange, C., DeMeo, D., Silverman, E. K., Weiss, S. T. \& Laird, N. M. PBAT: tools for family-based association studies. Am. J. Hum. Genet. 74, 367-369 (2004).

81 Laird, N. M., Horvath, S. \& Xu, X. Implementing a unified approach to family-based tests of association. Genet. Epidemiol. 19 (Suppl 1), S36-S42 (2000).

82 Deffenbacher, K. E., Kenyon, J. B., Hoover, D. M., Olson, R. K., Pennington, B. F., DeFries, J. C. et al. Refinement of the $6 \mathrm{p} 21.3$ quantitative trait locus influencing dyslexia: linkage and association analyses. Hum. Genet. 115, 128-138 (2004).
83 Meng, H., Smith, S. D., Hager, K., Held, M., Liu, J., Olson, R. K. et al. DCDC2 is associated with reading disability and modulates neuronal development in the brain. Proc. Natl Acad. Sci. USA 102, 17053-17058 (2005).

84 Schumacher, J., Anthoni, H., Dahdouh, F., Konig, I. R., Hillmer, A. M., Kluck, N. et al. Strong genetic evidence of DCDC2 as a susceptibility gene for dyslexia. Am. J. Hum. Genet. 78, 52-62 (2006).

85 Storey, J.D. A direct approach to false discovery rates. J. R. Stat. Soc. Ser. B 64, 479-498 (2002).

86 Benjamini, Y., Drai, D., Elmer, G., Kafkafi, N. \& Golani, I. Controlling the false discovery rate in behavior genetics research. Behav. Brain Res. 125, 279-284 (2001).

87 Wigg, K.G., Couto, J.M., Feng, Y., Anderson, B., Cate-Carter, T.D., Macciardi, F. et al. Support for EKN1 as the susceptibility locus for dyslexia on 15q21. Mol. Psychiatry 9, 1111-1121 (2004).

88 Benjamini, Y. \& Yekutieli, D. Quantitative trait loci analysis using the false discovery rate. Genetics 171, 783-790 (2005).

89 Mascheretti, S., Bureau, A., Battaglia, M., Simone, D., Quadrelli, E., Croteau, J. et al. An assessment of gene-by-environment interactions in developmental dyslexia-related phenotypes. Genes Brain Behav. 12, 47-55 (2013).

90 Plomin, R., Kovas, Y. \& Haworth, C. M. Generalist Genes: Genetic Links Between Brain, Mind, and Education. Mind Brain Educ. 1, 11-19 (2007).

Supplementary Information accompanies the paper on Journal of Human Genetics website (http://www.nature.com/jhg) 